\title{
TLR5/7-mediated PI3K activation triggers epithelial-mesenchymal transition of ovarian cancer cells through WAVE3-dependent mesothelin or OCT4/SOX2 expression
}

\author{
GA BIN PARK $^{1}$ and DAEJIN KIM ${ }^{2}$ \\ ${ }^{1}$ Department of Biochemistry, Kosin University College of Medicine, Busan 49267; \\ ${ }^{2}$ Department of Anatomy, Inje University College of Medicine, Busan 47392, Republic of Korea
}

Received March 8, 2017; Accepted August 1, 2017

DOI: $10.3892 /$ or.2017.5941

\begin{abstract}
Toll-like receptor (TLR)-mediated signaling induces cell migration or invasion in several tumors and various stages of cancer. Interactions of mesothelin, a 40-kDa cell surface glycoprotein, with cancer antigen 125 (CA125) is associated with drug resistance, metastasis, and poor clinical outcome of ovarian cancer patients. In this study, we examined the role of TLR5 and TLR7 in the metastasis of ovarian cancer through the induction of mesothelin/CA125 expression and investigated its underlying mechanism. TLR5 agonist (flagellin) and TLR7 agonist (imiquimod) upregulated mesenchymal phenotypes and produced epithelial-mesenchymal transition (EMT)-related cytokines in the SKOV3 cells; however, TLR7 expressing CaOV3 cells had no response to the specific ligand, imiquimod, for enhancing its EMT processes. Stimulation of the SKOV3 cells with flagellin or imiquimod activated Wiskott-Aldrich syndrome protein verprolin-homologous 3 (WAVE3) and mesothelin/CA125, whereas it suppressed the expression of TAp63. Moreover, knockdown of TLR5 or TLR7 in SKOV3 cells profoundly impaired the TLR5- or TLR7intiated downstream signaling pathway. Loss of WAVE3 in SKOV3 cells led to the inhibition of invasion, suppression of mesenchymal characteristics, prevention of OCT4/SOX2 secretion, and attenuation of mesothelin/CA125 expression after stimulation with flagellin or imiquimod. Although the disruption of mesothelin decreased the migratory activity of the TLR5/7-activated SKOV3 cells, knockdown of mesothelin failed to reduce the expression of mesenchymal markers, OCT4, and SOX2. In addition, targeting OCT4 or SOX2 with siRNA had no effect on the expression of mesothelin
\end{abstract}

Correspondence to: Dr Daejin Kim, Department of Anatomy, Inje University College of Medicine, Bokjiro-75, Busanjin-gu, Busan 47392, Republic of Korea

E-mail: kimdj@inje.ac.kr

Key words: Toll-like receptor, PI3K, epithelial-mesenchymal transition, TAp63, WAVE3, ovarian cancer and the suppression of transcriptionally active p63 (TAp63) in the TLR5/7-stimulated SKOV3 cells. Our results suggest that TLR5/7-mediated WAVE3 activation not only controls the mesothelin-related EMT processes but also modulates OCT4/SOX2-mediated mesenchymal marker expression. Taken together, both TLR5 and TLR7 expression are critical for the TLR5/7-induced metastasis of ovarian cancer and the inhibition of WAVE3 might be a new therapeutic target to control ovarian cancer metastasis.

\section{Introduction}

High mortality and poor prognosis due to late diagnosis are the main characteristics of ovarian cancer (1). Cancer metastasis is also considered a major cause of death in ovarian cancer patients (2). TLR stimulation in cancer cells is known to be closely related with proliferation and progression in various carcinomas (3). In addition, TLR activation promotes migration or invasion of several tumors (4-6). The expression of TLR2, TLR3, TLR4, and TLR5 are observed on the surface of normal ovary epithelium as well as benign and malignant tumor conditions (7). TLR1, TLR7, and TLR9 are also detected in malignant cells, but expressed at low level (7). Thus, studying TLR activation and related mechanism of cancer progression is critical for the regulation of ovarian cancer metastasis.

High mesothelin (MSLN) expression is one of characteristics of several human cancers, including ovarian cancer (8). Mesothelin plays an important role in cancer cell survival, migration, invasion and tumor progression $(9,10)$. Secreted mesothelin is capable of binding to CA125/MUC16 (11) and overexpression of CA125 on the surface of ovarian cancer cells is strongly associated with poor survival of ovarian cancer patients $(11,12)$. Mesothelin/CA125 binding promotes migration and invasion of pancreatic cancer cells via activation of matrix metalloproteinase 7 (MMP7) (13). Mesothelin triggers cancer cell survival, proliferation and drug resistance through phosphatidylinositol 3-kinase (PI3K)/Wnt/NF- $\mathrm{KB}$-signaling pathways (14). The PI3K/Akt pathway generally promotes the inhibition of apoptosis and progression of the cell cycle (15). TLRs stimulation is functionally associated with tumor growth and progression (16). We also reported that TLR4-mediated 
PI3K activation controls the invasion and metastasis of ovarian cancer through the production of galectin-1 (17). However, the underlying mechanism and association of PI3K for inducing mesothelin in TLR5/7-activated ovarian cancer cells remains unclear.

The WASP (Wiskott-Aldrich syndrome protein) and WAVE (WASP verprolin-homologous) families of proteins play important roles in the morphological changes and cytokinesis through the regulation of actin-cytoskeleton interactions (18). Although WAVE1 and WAVE2 are essential for the formation of the peripheral leading edge in invading fibroblasts (19), WAVE3 is also required for the process of epithelial mesenchymal transition (EMT) of various cancers, including breast, liver, and prostate (20-22). In addition, WAVE3 interacts with p85, the regulatory subunit of the PI3K, to activate the downstream signaling cascade (23). Although WAVE3 is one of the attractive diagnostic and therapeutic targets for anticancer therapy, the underlying mechanism of its induction and association with mesothelin in TLR-stimulated ovarian cancer remains poorly understood.

Ovarian cancer cells express a variety of TLRs (7). Furthermore, signaling and response induced by TLR5 activation regulates tumor growth and progression of ovarian cancer (24). Based on these studies, we hypothesized that the stimulation of TLR5 and TLR7 on the surface of ovarian cancer cells would induce PI3K/Akt-mediated epithelial-mesenchymal transition (EMT) through the regulation of several signaling pathways. We investigated the relationship between WAVE3 and mesothelin induction by the TLR5/7-activated PI3K/Akt signaling pathway. We also examined the association of TAp63 in TLR5/7-mediated ovarian cancer migration because induction of TAp63 expression is related to promotion of migratory and invasive activities in TLR4-mediated cancer cells (25).

\section{Materials and methods}

Cell lines and chemicals. The human ovarian cancer cell lines $\mathrm{CaOV} 3$ and SKOV3 were purchased from ATCC (Manassas, VA, USA). The CaOV3 cells were maintained in DMEM medium (Corning Inc., Corning, NY, USA) supplemented with $10 \%$ FBS (RMBIO, Missoula, MT, USA), penicillin, streptomycin, and glutamine at $37^{\circ} \mathrm{C}$ in $5 \% \mathrm{CO}_{2}$. The SKOV3 cells were maintained in RPMI-1640 medium (Corning Inc.) supplemented with $10 \% \mathrm{FBS}$, penicillin, streptomycin, and glutamine at $37^{\circ} \mathrm{C}$ in $5 \% \mathrm{CO}_{2}$. Flagellin (TLR5 ligand) was purchased from Sigma-Aldrich (St. Louis, MO, USA). Imiquimod (TLR7 ligand) was obtained from Santa Cruz Biotechnology (Santa Cruz, CA, USA). A66 (p110 $\alpha$ inhibitor), TGX-221 (p110 $\beta$ inhibitor), CAL-101 (p110 inhibitor), LY294002 (pan-p110 inhibitor), Bay 80-6946 (p110 $\alpha$ and p110 $\beta$ dual inhibitor), and pictilisib (p110 $\alpha$ and p110 dual inhibitor) were obtained from Selleck Chemicals (Houston, TX, USA).

Proliferation assay with Cell Counting Kit-8. Cell proliferation was measured using Cell Counting Kit-8 (CCK-8) (Enzo Life Sciences, Farmingdale, NY, USA) according to the manufacturer's instructions. The cells were seeded into $96-$ well plates $\left(2 \times 10^{4}\right.$ cells/well $)$ and treated with flagellin $(0.1 \mu \mathrm{g} / \mathrm{ml})$ or imiquimod (4 $\mu \mathrm{g} / \mathrm{ml})$. For comparison, non-treated control cells were cultured with media in the presence of DMSO. After $24 \mathrm{~h}$, the cells were stained with $10 \mu \mathrm{l}$ of CCK- 8 dye in $90 \mu \mathrm{l}$ of culture medium for $2 \mathrm{~h}$ at $37^{\circ} \mathrm{C}$. The absorbance was measured at $450 \mathrm{~nm}$.

Western blotting. The cells were washed in PBS and lysed in NP-40 buffer (Elpis Biotech, Daejeon, Korea) supplemented with a protease inhibitor cocktail (Sigma-Aldrich). Protein phosphorylation states were preserved by the addition of phosphatase inhibitors (Cocktail II, Sigma-Aldrich) to the NP-40 buffer. Protein concentrations were determined by using a BCA assay kit (Pierce, Rockford, IL, USA). The proteins $(10 \mu \mathrm{g} / \mathrm{sample})$ were resolved by SDS-PAGE and then transferred onto a nitrocellulose membrane (Millipore Corp., Billerica, MA, USA). The membranes were blocked with 5\% non-fat milk prior to western blot analysis. Chemiluminescence was detected using an ECL kit (Advanta Corp., Menlo Park, CA, USA) and the Amersham Imager 600 (GE Healthcare Life Sciences, Little Chalfont, UK). The following primary Abs were used: E-cadherin, N-cadherin, Slug, Snail, Vimentin, TCF8/Zeb1, $\beta$-actin, MMP2, MMP9, MyD88, p110 $\alpha$, p110 $\beta$, p1108, WAVE3, mesothelin, SOX2, and OCT4 (Cell Signaling Technology, Beverly, MA, USA); $\alpha$-SMA (Bioss, Woburn, MA, USA); CA125 (Abcam, Cambridge, UK); TAp63 (BioLegend, San Diego, CA, USA); TLR5 and TLR7 (Santa Cruz Biotechnology).

Small interfering RNA (siRNA) transfection. Experimentally verified human WAVE3-small interfering RNA (siRNA) duplex, human OCT4-siRNA duplex, human SOX2siRNA duplex, human mesothelin-siRNA duplex, human TLR5-siRNA duplex, human TLR7-siRNA duplex, and negative control-siRNA were obtained from Bioneer Corp. (Daejeon, Korea). Cells were seeded at a concentration of $1 \times 10^{5}$ per well in a 6-well plate and grown overnight. The cells were then transfected with $200 \mathrm{nM}$ siRNA using Lipofectamine RNAiMAX Reagent (Invitrogen, Carlsbad, CA, USA) according to the manufacturer's instructions. The cells were used for further experiments at $36 \mathrm{~h}$ after transfection and then treated with TLR agonists for $24 \mathrm{~h}$.

Quantification of human cytokines by ELISA. The concentrations of active TGF- $\beta 1$, TNF- $\alpha$, VEGF, and IL- 8 in the cell culture supernatants were quantified by single cytokine ELISA assay kits (R\&D Systems, Minneapolis, MN, USA). Mesothelin was quantified with a kit from BioLegend. WAVE3, SOX2, and OCT4 were quantified with kits from MyBioSource (San Diego, CA, USA). The data are expressed as the average value from a number of biological replicates \pm standard deviation (SD).

Invasion assay. The invasion assay was performed using the CultreCoat 96-well Medium BME Cell Invasion Assay kit (R\&D Systems) according to the manufacturer's protocol. Cells $\left(2.5 \times 10^{4}\right)$ in serum-free RPMI-1640 or DMEM containing $0.1 \%$ FBS were seeded into the upper chamber and the lower compartment was filled with RPMI-1640 or DMEM containing $10 \% \mathrm{FBS}$ as the chemoattractant. After incubation for $24 \mathrm{~h}$, the non-invading cells on the upper membrane 
A

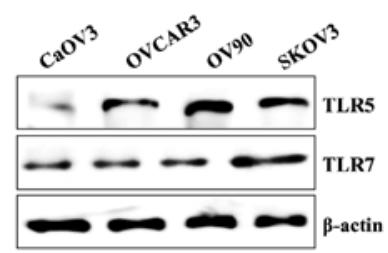

B

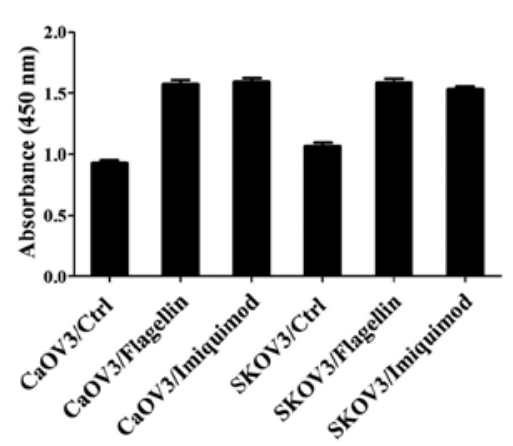

C

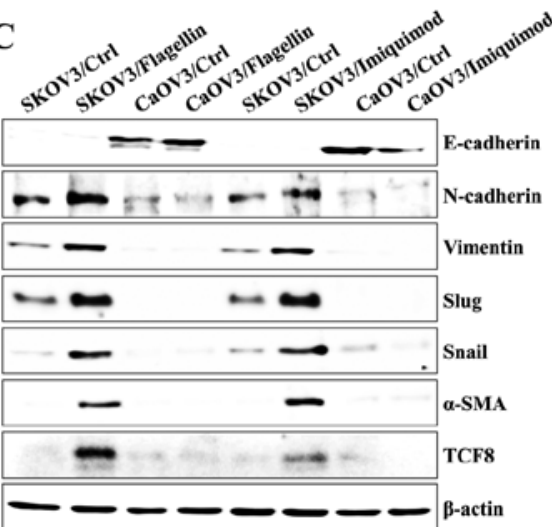

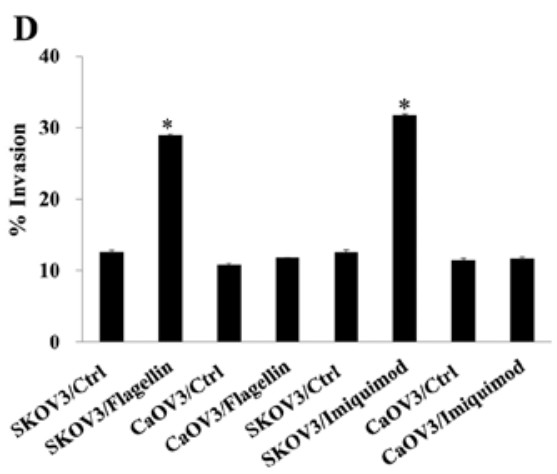
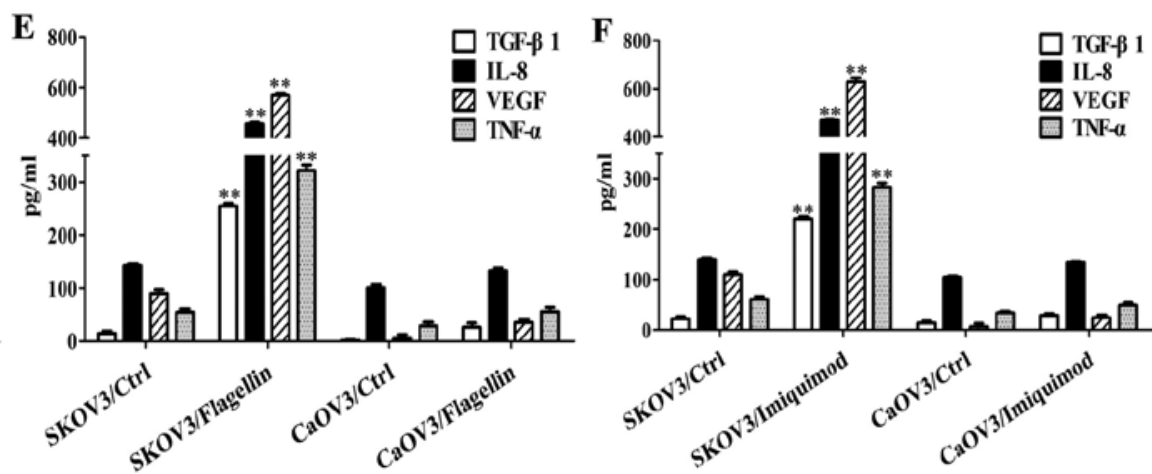

Figure 1. TLR5/7 agonist increases the invasion and secretion of EMT-related cytokines of ovarian cancer cells. (A) The protein levels of TLR5/7 on ovarian cancer cells were measured using western blotting. $\beta$-actin was used as a loading control. (B) Effect of TLR agonists on the proliferation of CaOV3 and SKOV3 cells. The cells were seeded into 96 -well plates $\left(2 \times 10^{4}\right.$ cells/well) and treated with flagellin $(0.1 \mu \mathrm{g} / \mathrm{ml})$ or imiquimod $(4 \mu \mathrm{g} / \mathrm{ml})$. After incubation for $24 \mathrm{~h}$, cell proliferation was determined using the Cell Counting Kit-8 (CCK-8) according to the manufacturer's instructions. (C-F) Cells (1.5x10 $/$ well) were seeded into 6 -well plates and grown overnight. The cells were cultured with or without TLR agonists $(0.1 \mu \mathrm{g} / \mathrm{ml}$ flagellin or $4 \mu \mathrm{g} / \mathrm{ml}$ imiquimod) for $24 \mathrm{~h}$. (C) EMT markers (E-cadherin, N-cadherin, Vimentin, Slug, Snail, $\alpha$-SMA, and TCF8) were determined using western blotting. (D) The invasiveness of the SKOV3 cells was enhanced by TLR agonists (flagellin or imiquimod) as determined with the BME cell invasion assay described in Materials and methods. Each value is the mean \pm standard deviation of 3 determinations. ${ }^{*} \mathrm{P}<0.01$. (E and F) Culture supernatants were collected $24 \mathrm{~h}$ after stimulation with TLR agonists (flagellin or imiquimod), and the amounts of active TGF- $\beta 1$, IL-8, VEGF, and TNF- $\alpha$ were determined by ELISA. Data are presented as the mean of three independent experiments, and the error bars represent $\mathrm{SD}$ of the means. ${ }^{* *} \mathrm{P}<0.005$. The results are representative of three independent experiments.

surface were removed by wiping with a cotton swab. The invaded cells were stained with calcein-AM and quantified using a microplate reader.

Statistical analyses. Data are expressed as the mean \pm standard deviation (SD). Statistical analysis was conducted using one-way analysis of the variance. A P-value $<0.05$ was considered to indicate a statistically significant difference.

\section{Results}

Binding to TLR5/7 induces changes in SKOV3 cell motility and secretion of EMT-related cytokines. We first examined the expression of TLR5 and TLR7 to determine the effect of TLR stimulation on EMT processes in ovarian cancer cells. First, we selected the four cell lines (CaOV3, OVCAR3, OV90 and SKOV3) as model of ovarian cancer (26) and checked the expression of TLR5 and TLR7 as well as the proliferation after stimulation with TLR5 ligand (flagellin) or TLR7 ligand (imiquimod). Although TLR7 was detected in all ovarian cancer cell lines, the appearance of TLR5 on CaOV3 cells was very weak compared to the other cell lines (Fig. 1A). TLR5 or TLR7 activation by specific agonist had no effect on the proliferation of $\mathrm{CaOV} 3$ and SKOV3 cells (Fig. 1B). Based on those results, we selected $\mathrm{CaOV} 3$ cells as the representative of primary ovarian cancer and SKOV3 cells as the model of metastatic ovarian cancer (27). Treatment with flagellin and imiquimod of SKOV3 cells not only induced the mesenchymal markers (N-cadherin, Vimentin, Slug, Snail, $\alpha$-SMA, and TCF8) but also reduced the epithelial marker, E-cadherin; however, the stimulation of CaOV3 cells with TLRs had no influence on upregulation of the mesenchymal phenotype even after stimulation with flagellin and imiquimod (Fig. 1C). The invasive activity of the SKOV3 cells was also enhanced by stimulation with flagellin or imiquimod (Fig. 1D). The SKOV3 cells significantly increased the EMT-related cytokines (TGF- $\beta 1$, IL-8, VEGF, and TNF- $\alpha$ ) compared with nontreated control cells after treatment with flagellin (Fig. 1E) or imiquimod (Fig. 1F). These results suggest that activation of TLR $5 / 7$ might be one of the critical targets to modulate ovarian cancer metastasis and invasion.

TLR5/7 stimulation induces the expression of EMT-associated signaling molecules in SKOV3 cells. Next, we examined the signaling pathway that is influenced by the activation of TLR5/7 responsible for the migration and invasion in ovarian 

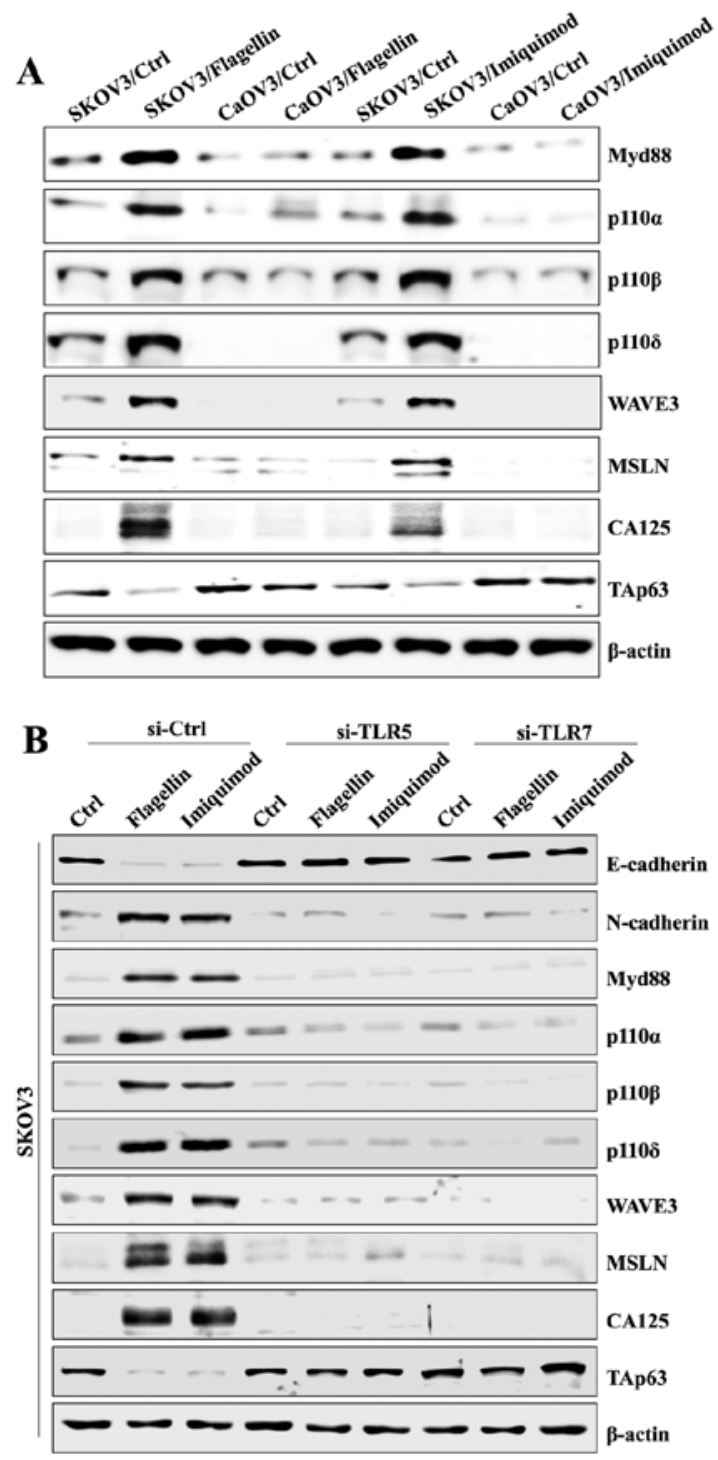

C

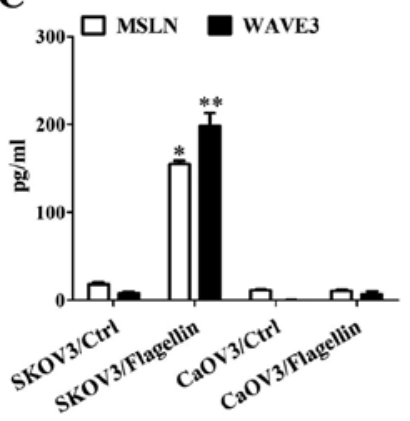

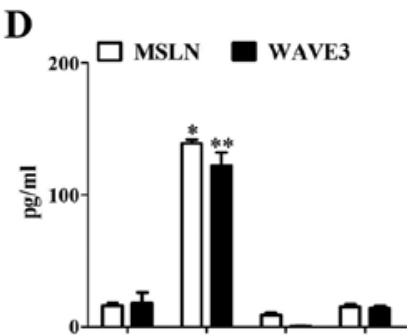

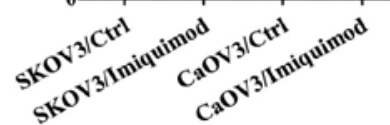

$\mathbf{E}$

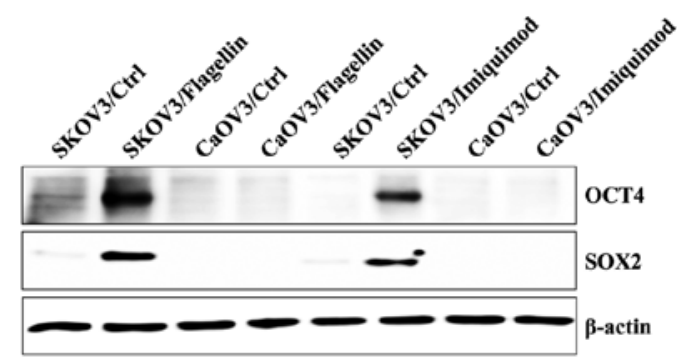

F

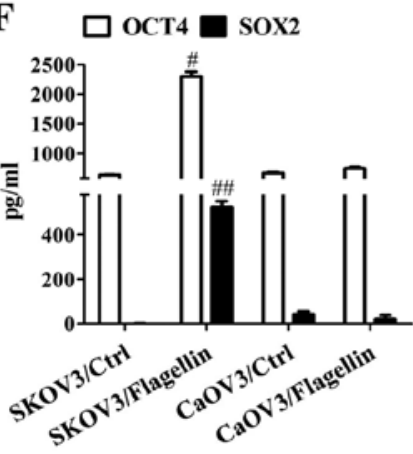

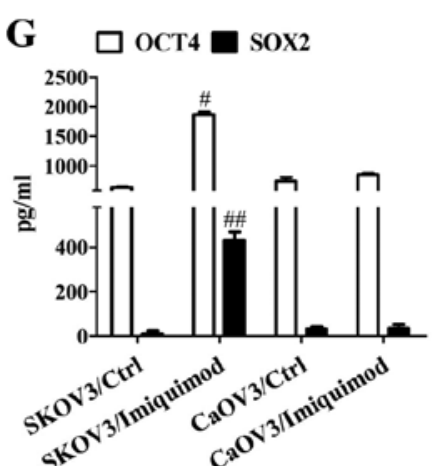

Figure 2. TLR5/7 stimulation induces the expression of EMT-associated signaling molecules in ovarian cancer cells. (A-G) Cells (1.5x105/well) were seeded into 6-well plates and grown overnight. The cells were cultured with or without TLR agonists $(0.1 \mu \mathrm{g} / \mathrm{ml}$ flagellin or $4 \mu \mathrm{g} / \mathrm{ml}$ imiquimod) for $24 \mathrm{~h}$. (B) To determine the effect of TLR5 or TLT7 on EMT-related signal transduction, the cells were seeded into 6-well plates (1x105/well) and transfected with siRNA oligonucleotides of TLR5-siRNA (200 nM) or TLR7-siRNA (200 nM) or control-siRNA for $36 \mathrm{~h}$ and then treated with TLR agonists for $24 \mathrm{~h}$. (A, B and E) Total cell lysates were immunoblotted with the indicated antibodies. $\beta$-actin served as the loading control. (C, D, F and G) The culture supernatants were collected $24 \mathrm{~h}$ after stimulation with flagellin (C and F) or imiquimod ( $\mathrm{D}$ and $\mathrm{G}$ ), and then the amounts of mesothelin, WAVE3, SOX2, and OCT4 were determined by ELISA. Data are presented as the mean of three independent experiments, and the error bars represent $\mathrm{SD}$ of the means. ${ }^{*} \mathrm{P}<0.001 .{ }^{* *} \mathrm{P}<0.05$. ${ }^{\#} \mathrm{P}<0.005$. ${ }^{\# \#} \mathrm{P}<0.01$. The results are representative of three independent experiments. MSLN, mesothelin.

cancer cells. The TLR5/7-activated ovarian cancer cells showed activation of MyD88 expression; the levels of PI3K $\mathrm{p} 110 \alpha, \beta$, and $\delta$ isoforms were significantly increased in the TLR5/7-stimulated SKOV3 cells compared to the non-activated cells (Fig. 2A). Although the expression of TAp63 was inhibited, the levels of WAVE3, mesothelin, and CA125 were upregulated in the TLR5/7-treated SKOV3 cells (Fig. 2A). However, gene silencing of TLR5 or TLR7 with siRNA profoundly blocked the expression of mesenchymal marker (N-cadherin) and prevented the activation of downstream signaling pathway in TLR5- or TLR7-stimulated SKOV3 cells (Fig. 2B). The secretion of mesothelin and WAVE3 by SKOV3 cells was detected after stimulation with TLR5 and TLR7 (Fig. 2C and D). The TLR5/7-activated SKOV3 cells showed an induction in the expression of SRY-related
HMG-box gene 2 (SOX2) and Octamer-binding transcription factor 4 (OCT4) proteins (Fig. 2E), which contribute to cancer invasion $(28,29)$. In addition, secreted SOX 2 and OCT4 were detected in the culture supernatant (Fig. 2F and G). These results suggest that TLR5/7 responses to their ligands play a major role in triggering the EMT-related signal transduction of metastatic ovarian cancer cells.

TLR5/7-dependent PI3K activation regulates WAVE3mediated cancer cell motility. Previously, we found that TLR stimulation contributed to the activation of PI3K catalytic subunits. Thus, the biological significance of PI3K in cancer cell metastasis and its effect on the EMT-related signaling molecules were examined using specific pharmacological inhibitors. Specific inhibitor against PI3K p110 $\alpha, \beta$, and $\delta$ 
$\mathbf{A}$

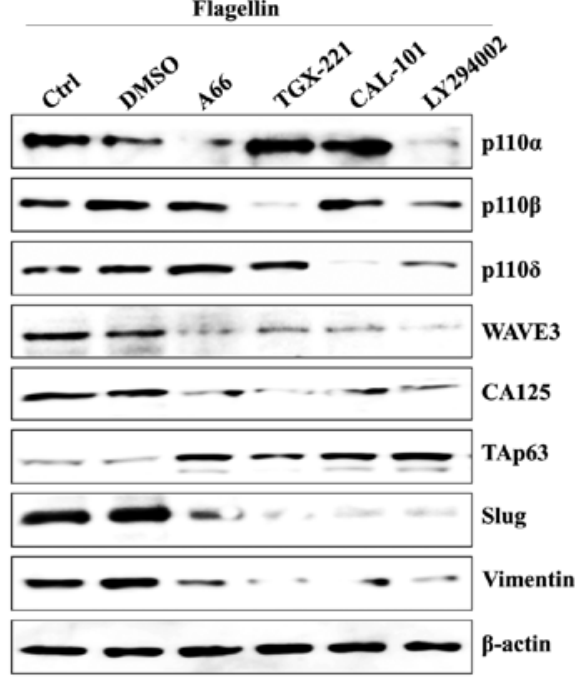

C

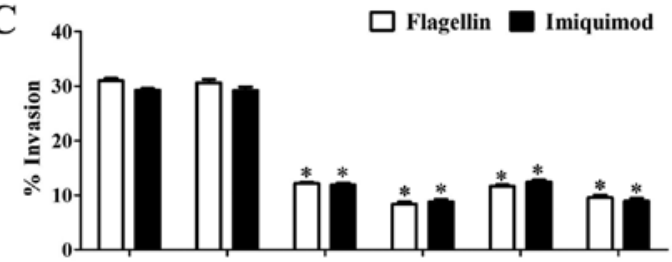

D

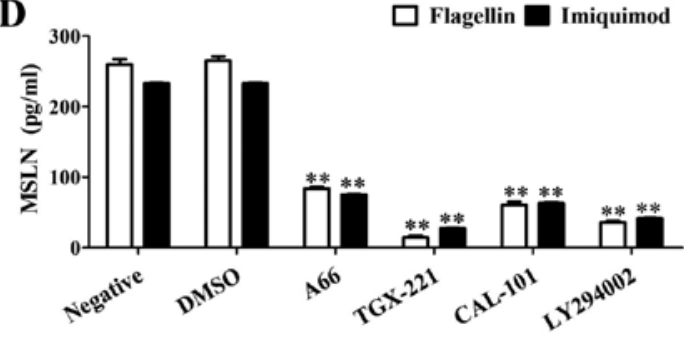

B

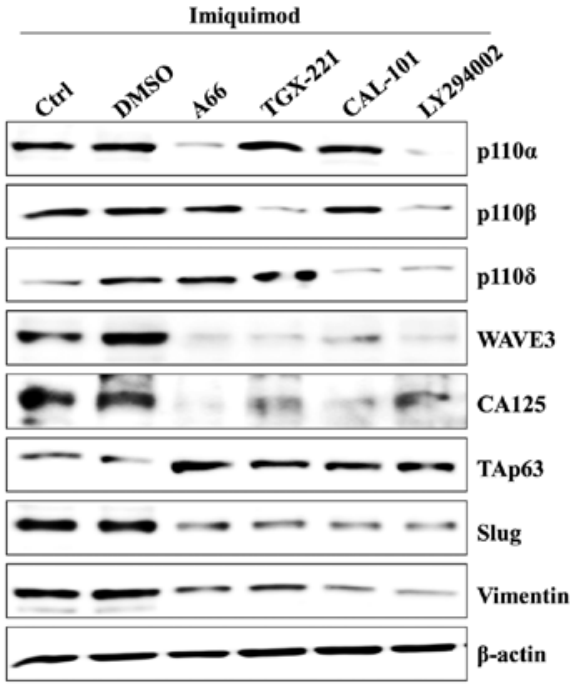

$\mathbf{E}$

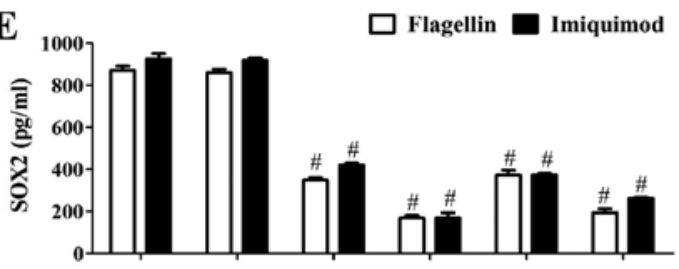

$\mathbf{F}$

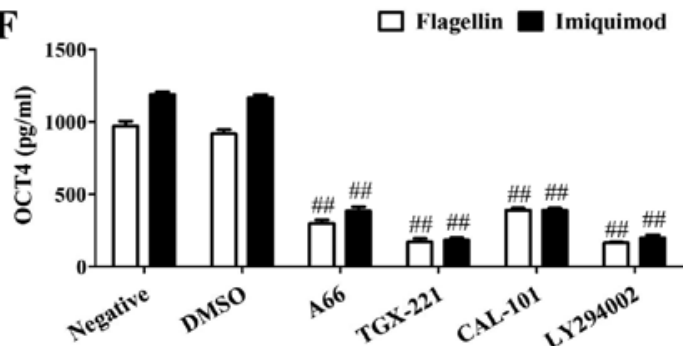

Figure 3. TLR5/7-dependent PI3K activation regulates WAVE3-mediated cancer cell motility. Cells (1.5x105/well) were seeded into 6-well plates and incubated overnight. The cells were pre-incubated with $50 \mu \mathrm{M}$ A66, $50 \mu \mathrm{M}$ TGX-221, $50 \mu \mathrm{M}$ CAL-101, $50 \mu \mathrm{M}$ LY294002, $200 \mathrm{nM}$ BAY 80-6946, or 200 nM pictilisib for $2 \mathrm{~h}$ and then treated with TLR agonists $(0.1 \mu \mathrm{g} / \mathrm{ml}$ flagellin or $4 \mu \mathrm{g} / \mathrm{ml}$ imiquimod) for $24 \mathrm{~h}$. (A and B) Total cell lysates were immunoblotted with the indicated antibodies. (C) The invasiveness of the TLR5/7-stimulated SKOV3 cells was blocked by PI3K inhibitors as determined by the BME cell invasion assay described in Materials and methods. Each value is the mean $\pm \mathrm{SD}$ of 3 determinations. ${ }^{*} \mathrm{P}<0.005$. (D-F) The culture supernatants were collected $36 \mathrm{~h}$ after transfection, and the amounts of mesothelin (D), SOX2 (E), and OCT4 (F) were determined by ELISA. Data are presented as the mean of three independent experiments, and the error bars represent $\mathrm{SD}$ of the means. ${ }^{* *} \mathrm{P}<0.005$. ${ }^{\#} \mathrm{P}<0.01$. ${ }^{\# \#} \mathrm{P}<0.01$. The results are representative of three independent experiments. MSLN, mesothelin.

isoforms considerably suppressed the induction of WAVE3 and CA125 in the SKOV3 cells after treatment with flagellin or imiquimod, whereas the levels of TAp63 were upregulated (Fig. 3A and B). Additionally, the specific inhibition of each PI3K p110 isoform efficiently reversed the expression of TAp63 and reduced the mesenchymal properties of the TLR5/7activated SKOV3 cells (Fig. 3A and B). Pharmacological inhibition of PI3K p110 isoform significantly prevented cell motility (Fig. 3C), secretion of mesothelin (Fig. 3D) and production of OCT4 (Fig. 3E) and SOX2 (Fig. 3F) in the TLR5/7-treated SKOV3 cells. These results suggest that the PI3K-mediated the expression of WAVE3, mesothelin, SOX2, and OCT4 activates migration and invasion capacity of the ovarian cancer cells after stimulation with flagellin or imiquimod.

WAVE3 is required for the expression of mesothelin/CA125 and production of OCT4/SOX2 in the TLR5/7-mediated invasion activity of SKOV3 cells. Next, we investigated the relationship of EMT-related molecules to better understand how the TLR5/7-mediated signaling pathway regulates cancer cell motility. In flagellin- or imiquimod-treated SKOV3 cells, the knockdown of WAVE3 using siRNA resulted in the loss of expression of mesothelin and CA125; however, the level of TAp63 was not downregulated by stimulation with flagellin or imiquimod in the WAVE3-silenced SKOV3 cells (Fig. 4A). Silencing of the WAVE3 in the SKOV3 cells blocked the expression of mesenchymal markers, matrix metalloproteinase 2 (MMP2) and MMP9 (Fig. 4B) and inhibited the invasive activity of the cells after TLR5/7 stimulation (Fig. 4C and D). In addition, downregulation of WAVE3 markedly suppressed the expression (Fig. 4E) and secretion of OCT4 and SOX2 in the TLR5/7-activated SKOV3 cells (Fig. 4F and G). Although WAVE3-silencing reduced the expression of mesothelin and CA125 along with the activation of MMP2/MMP9 in the TLR5/7-stimulated SKOV3 cells, silencing of the mesothelin 

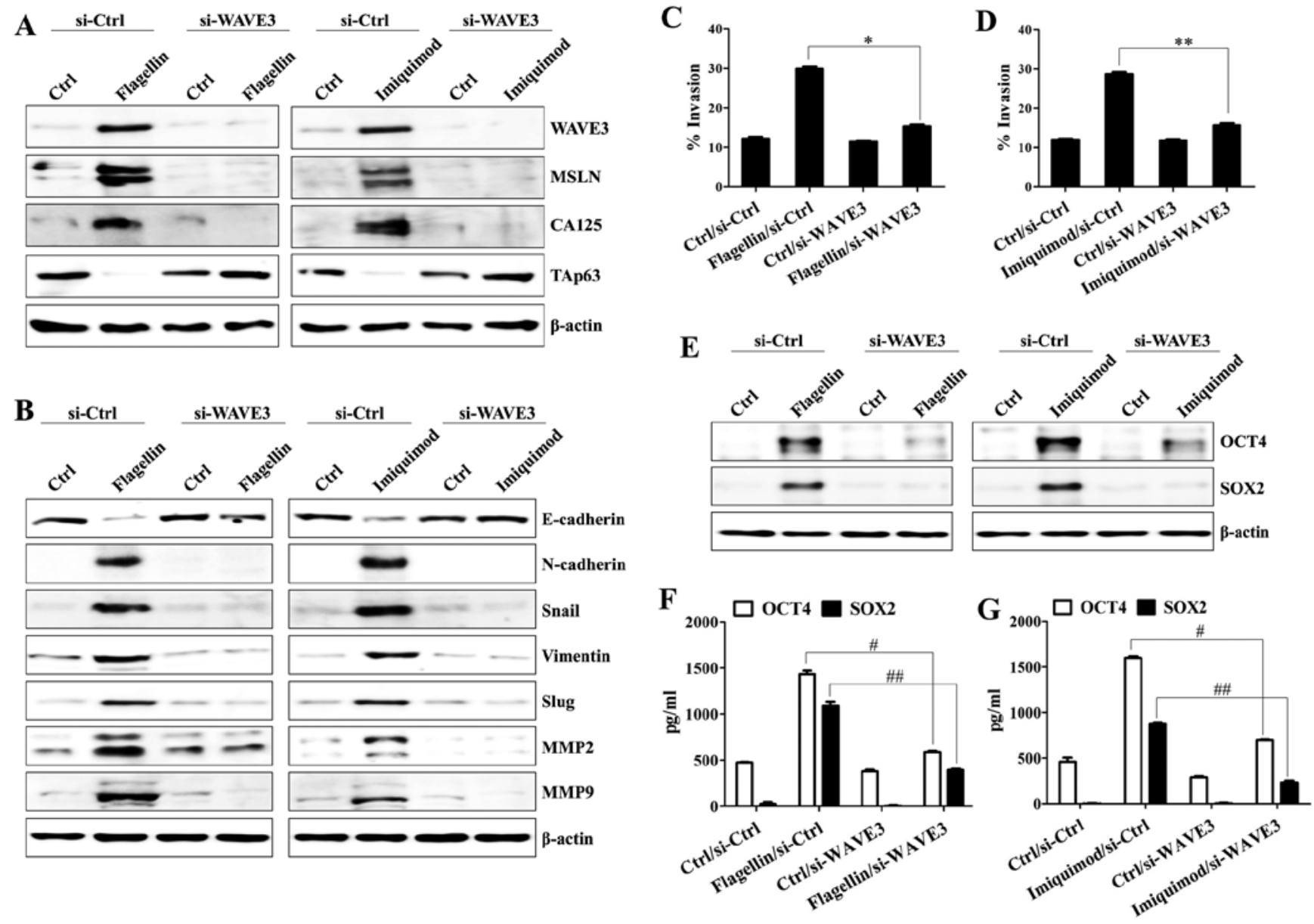

Figure 4. WAVE3 is required for the expression of mesothelin/CA125 and production of OCT4 and SOX2 in TLR5/7-mediated EMT processes in ovarian cancer cells. The SKOV3 cells were seeded into 6-well plates (1.5x10\% $/$ well), transfected with WAVE3-siRNA (200 nM) or control-siRNA for $36 \mathrm{~h}$ and then treated with TLR agonists $(0.1 \mu \mathrm{g} / \mathrm{ml}$ flagellin or $4 \mu \mathrm{g} / \mathrm{ml}$ imiquimod) for $24 \mathrm{~h}$ prior to the experiments. (A, B and E) Total cell lysates were immunoblotted with the indicated antibodies. $\beta$-actin served as the loading control. (C and D) The invasiveness of the TLR5/7-stimulated SKOV3 cells was inhibited by WAVE3 silencing as determined by the BME cell invasion assay described in Materials and methods. Each value is the mean \pm SD of 3 determinations. "P<0.01. ${ }^{* *} \mathrm{P}<0.01$. ( $\mathrm{F}$ and $\left.\mathrm{G}\right)$ The culture supernatants were collected $60 \mathrm{~h}$ after transfection and then stimulation with TLR agonists, and the amounts of secreted SOX2 and OCT4 were determined by ELISA. Data are presented as the mean of three independent experiments, and the error bars represent SD of the means. ${ }^{\#} \mathrm{P}<0.01 .{ }^{\# \#} \mathrm{P}<0.01$. The results are representative of three independent experiments. MSLN, mesothelin.

gene using siRNA decreased only the level of CA125 in flagellin- or imiquimod-treated SKOV3 cells (Fig. 5A) but not the levels of WAVE3 or TAp63 (Fig. 5A). The expression of mesenchymal markers, OCT4, and SOX2 were not affected by silencing of mesothelin gene in the flagellin- or imiquimod-treated SKOV3 cells (Fig. 5B), whereas the targeted inhibition of mesothelin reduced the expression of MMP2 and MMP5 after the stimulation with TLR5 and TLR7 ligands (Fig. 5B). Stimulation with flagellin or imiquimod of the mesothelin-silenced SKOV3 cells resulted in the attenuation of their migratory activity (Fig. 5C and D); however, the SKOV3 cells lacking mesothelin expression failed to decrease the production of SOX2 and OCT4 after treatment with flagellin or imiquimod (Fig. 5E and F). These results suggest that WAVE3 not only plays a critical role in the mesothelin/ CA125-mediated TAp63 downregulation and MMP upregulation but also controls the expression of OCT4 and SOX2 in a mesothelin-independent manner.

OCT4 and SOX2 modulate the expression of mesenchymal markers in the TLR5/7-activated SKOV3 cells. Next, we inves- tigated whether the level of OCT4 or SOX2 has an effect on the expression of mesenchymal markers in the TLR5/7-stimulated SKOV 3 cells. The targeted inhibition of OCT4 or SOX2 using small interference RNA had no effect on the mesothelin upregulation and TAp63 downregulation of the flagellin- or imiquimod-treated SKOV3 cells (Fig. 6A). Moreover, silencing of OCT4 or SOX2 genes reduced the expression of $\mathrm{N}$-cadherin and Snail in SKOV3 after treatment with TLR5 and TLR7 ligands (Fig. 6A). In addition, the downregulation of OCT4 or SOX2 significantly suppressed the expression of activated MMP2 and MMP9 in the flagellin- or imiquimod-treated SKOV3 cells. These results suggest that the TLR5/7-mediated WAVE3 signaling pathway controls the EMT processes through the regulation of OCT4 and SOX2 expression.

\section{Discussion}

TLR activation in cancer cells promotes metastasis of cancer cells at various stages through the regulation of cell adhesion, invasion, angiogenesis, and increase in vascular permeability (3-6). The activation of TLR2:TLR6 complexes 
A

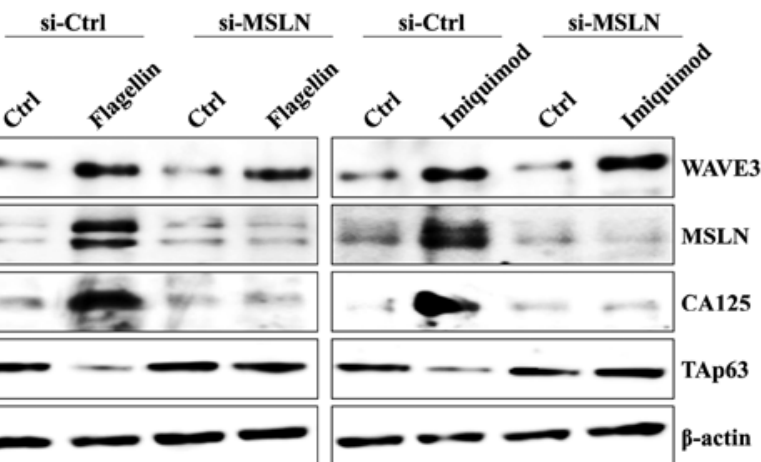

C
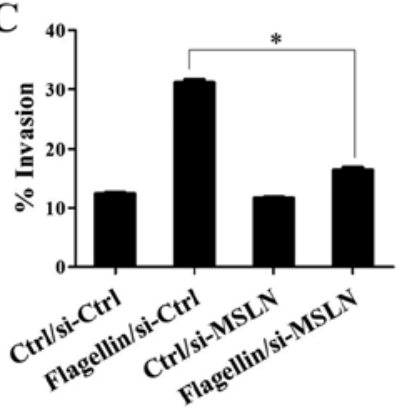

D

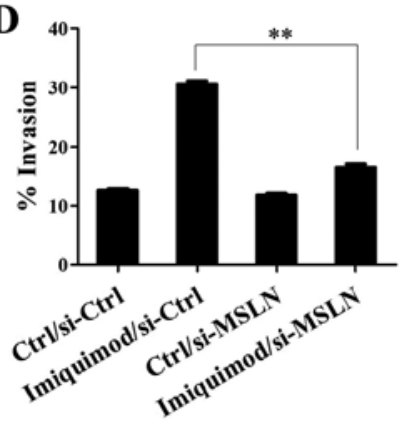

B

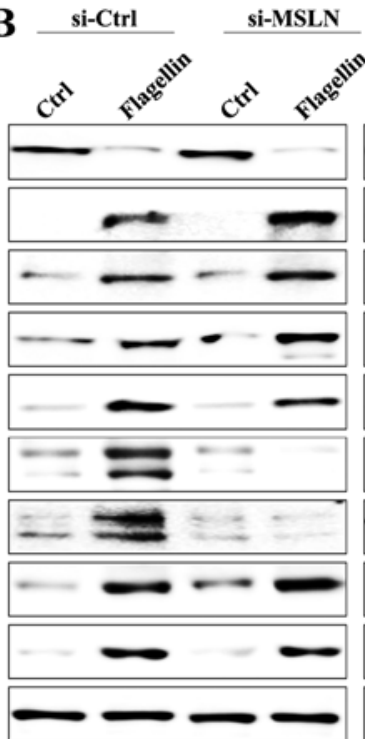

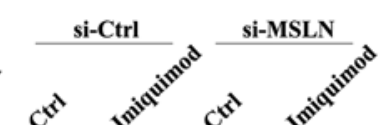

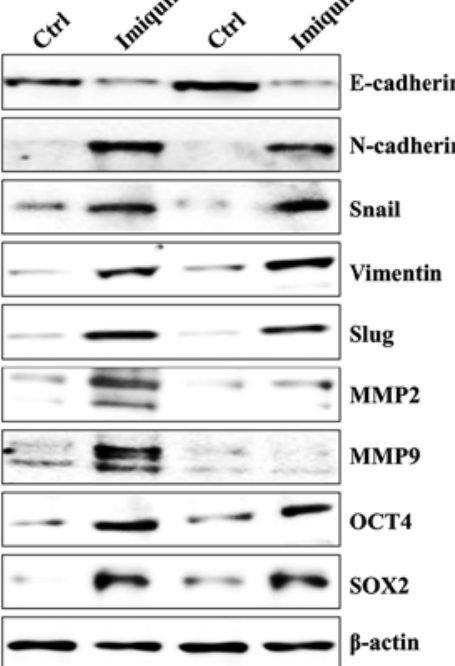

$\mathbf{E}$

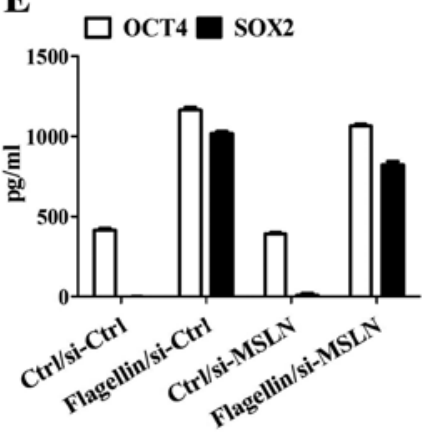

$\mathbf{F}$

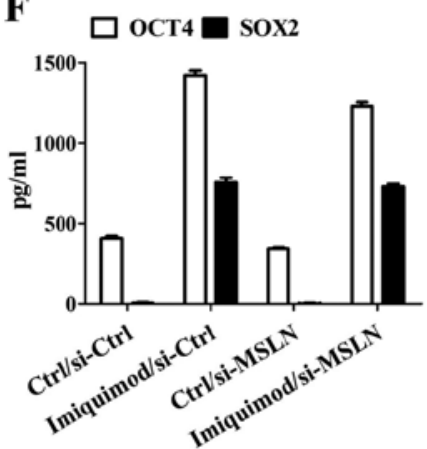

Figure 5. Mesothelin is required for invasiveness and production of OCT4 and SOX2 in the TLR5/7-mediated ovarian cancer EMT processes. The SKOV3 cells were seeded into 6-well plates ( $1.5 \times 10^{5} /$ well), transfected with mesothelin-siRNA ( $\left.200 \mathrm{nM}\right)$ or control-siRNA for $36 \mathrm{~h}$ and then treated with TLR agonists $(0.1 \mu \mathrm{g} / \mathrm{ml}$ flagellin or $4 \mu \mathrm{g} / \mathrm{ml}$ imiquimod) for $24 \mathrm{~h}$ prior to the experiments. (A and B) Total cell lysates were immunoblotted with the indicated antibodies. $\beta$-actin served as the loading control. (C and D) The invasiveness of the TLR5/7-stimulated SKOV3 cells was inhibited by mesothelin silencing as determined by the BME cell invasion assay described in Materials and methods. Each value is the mean \pm SD of 3 determinations. ${ }^{*} \mathrm{P}<0.01{ }^{. *} \mathrm{P}<0.01$. (E and $\mathrm{F}$ ) Culture supernatants were collected $60 \mathrm{~h}$ after transfection and then stimulation with TLR agonists, and the amounts of secreted SOX2 and OCT4 were determined by ELISA. Data are presented as the mean of three independent experiments, and the error bars represent SD of the means. The results are representative of three independent experiments. MSLN, mesothelin.

has been shown to enhance the metastatic growth of lung cancer by inducing TNF- $\alpha$ secretion in a rat model (4). The exposure of TLR4 ligands to lipopolysaccharides (LPS), induces cancer growth and lung metastasis of breast cancer by increasing angiogenesis (5). CpG oligonucleotide treatment decreased the expression of tissue inhibitor of metalloproteinase- 3 and increased levels of active MMP13 in TLR9-expressing breast cancer cells (6). TLR4 stimulation is also involved in ovarian cancer growth and sensitivity to apoptosis mediated by drugs or immune cells (30). Although TLR5 and TLR7 are detected on the ovarian epithelium under benign conditions and epithelial tumors (7), the role and signaling mechanism of TLR5 and TLR7 in ovarian cancer metastasis has yet to be determined.

In this study, SKOV3 cells, as representatives of metastatic ovarian cancer, strongly expressed TLR5 and TLR7, whereas the $\mathrm{CaOV} 3$ cells expressed TLR7, but their expression of TLR5 was weak. TLR5/7-dependent signaling contributed to the enhancement of cell motility through PI3K-mediated WAVE3 activation. Moreover, WAVE3 modulated the expression of mesothelin/CA125, downregulated the expression of TAp63, and promoted the expression of OCT4/SOX2-mediated mesenchymal markers in SKOV3 (Fig. 6B). Although TLR7 was also expressed in the $\mathrm{CaOV} 3$ cells, stimulation of these cells with imiquimod failed to activate downstream signaling pathways. Furthermore, downregulation of TLR5 or TLR7 by transfection with siRNA in SKOV3 cells prevented the initiation of TLR5- or TLR7-mediated signaling pathway. Our findings suggest that both TLR5 and TLR7 expression of ovarian cancer play critical roles in triggering WAVE3-dependent EMT processes.

Mesothelin, a plasma membrane differentiation antigen, is expressed at significantly high levels in several human cancers, including nearly all mesotheliomas (8) and approximately $70 \%$ of ovarian cancers (11). High mesothelin expression is positively correlated with drug resistance and short disease-free survival in ovarian cancer patients (31). Although mesothelin fails to provide diagnostic information about the cancer due to its low sensitivity and specificity as a tumor marker (32), the co-expression of CA125 and mesothelin is strongly correlated with induction of cancer cell metastasis and unfavorable 
$\mathbf{A}$

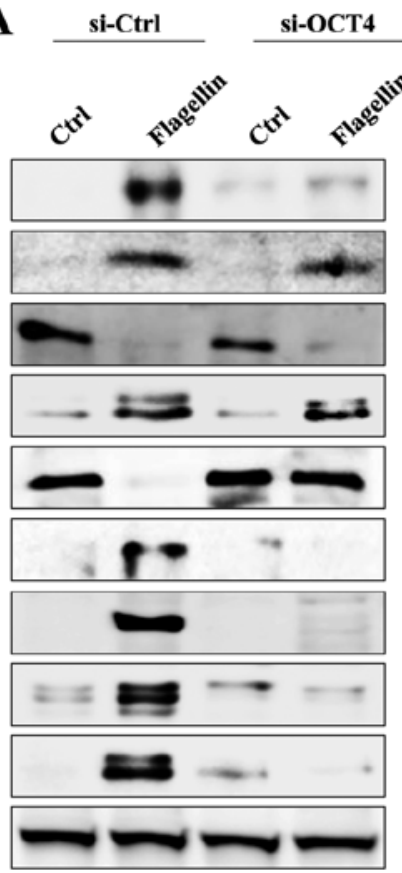

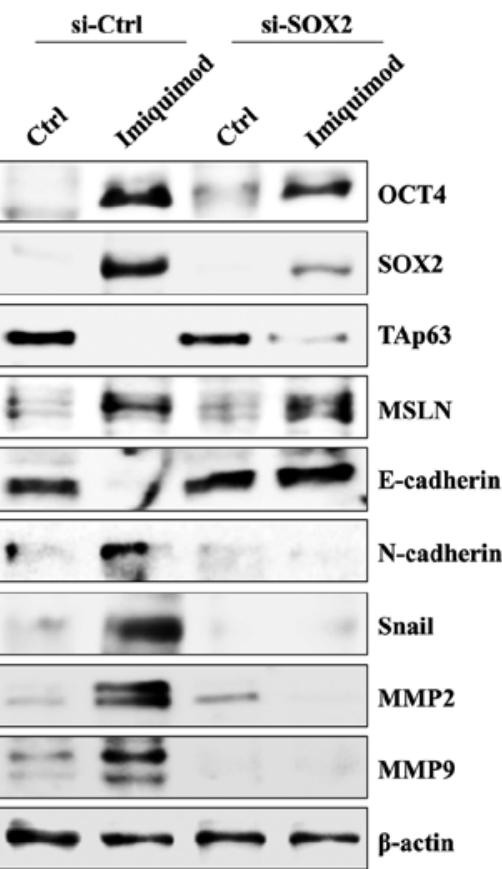

B

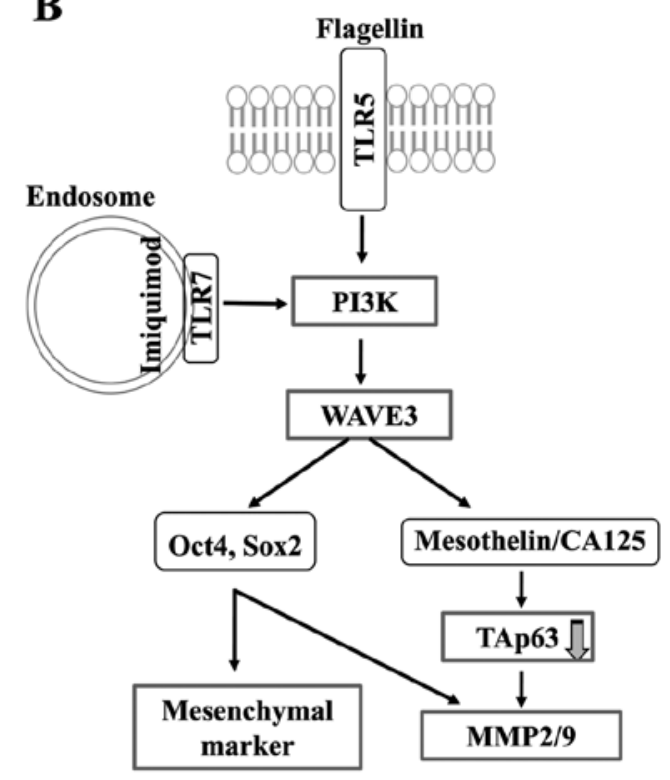

Figure 6. OCT4 and SOX2 modulate the expression of mesenchymal markers in the TLR5/7-activated SKOV3. (A) The SKOV3 cells were seeded into 6-well plates $\left(1.5 \times 10^{5} /\right.$ well), transfected with OCT4-siRNA $(300 \mathrm{nM})$, SOX2-siRNA $(300 \mathrm{nM})$ or control-siRNA for $36 \mathrm{~h}$ and then treated with TLR agonists $(0.1 \mu \mathrm{g} /$ $\mathrm{ml}$ flagellin or $4 \mu \mathrm{g} / \mathrm{ml}$ imiquimod) for $24 \mathrm{~h}$ prior to experiments. Total cell lysates were immunoblotted with the indicated antibodies. $\beta$-actin served as the loading control. (B) Schematic diagram of the intracellular signaling mechanism during TLR agonist (flagellin or imiquimod)-induced TLR5/7 activation of human ovarian cancer cells. Interaction of flagellin or imiquimod with TLR5 or TLR7 on SKOV3 cells transduces cellular signals through a common pathway that leads to the expression of p110 $\alpha \beta \delta$ and WAVE3. The subsequent secretion of SOX2, OCT4, and mesothelin by the stimulation of TLR5/7 expression and downregulation of TAp63 expression by mesothelin-CA125 interaction results in EMT processes, which induce metastasis of ovarian cancer cells. This study suggest that the metastasis of ovarian cancer cells may be due to increase in the expression of p110 $\alpha \beta$ and WAVE3 and the production of SOX2, OCT4, and mesothelin. MSLN, mesothelin.

patient outcomes in pancreatic cancer $(13,33)$. The molecular mechanisms that contribute to the induction of mesothelin and CA125 expression remain largely unknown. Furthermore, additional studies on mesothelin induction are still needed to define its role as a diagnostic marker for determining the clinical outcome and response to anti-cancer therapy. The levels of mesothelin and CA125 were upregulated after stimulation of the SKOV3 cells with flagellin or imiquimod. In addition, the loss of WAVE3 expression led to the complete downregulation of mesothelin and CA125 in the TLR5/7-activated SKOV3 cells. Our data suggest that the acquisition of WAVE3 activation is one of important steps in controlling the expression of mesothelin/CA125.

The WASP/WAVE proteins are closely related to cell shape changes, cytokinesis, and cell motility, through the re-organization of cytoskeletal proteins (34). The WAVE subfamily of proteins contains three members, WAVE1, WAVE2, and WAVE3 (35). WAVE3 has been shown to regulate motility and invasiveness of breast cancer cells through the activation of p38 and MMP (36). Knockdown of WAVE3 in cells results in the inhibition of NF- $\kappa \mathrm{B}$ signaling and activation of MMP9 (37). Treatment of cells with LY294002, an inhibitor of PI3K, also prevents WAVE3-mediated breast cancer cell migration (23). Although PI3K/Akt activation is involved in multiple signal transduction pathways (38), an important question that remains to be answered is whether the TLR5/7-mediated cancer cell migration is also regulated by the PI3K/WAVE3 pathway. TLR5/7-dependent PI3K activation led to the WAVE3-mediated expression of mesothelin and CA125 and the production of OCT4 and SOX2. Pharmacological inhibition of PI3K p110 isoform suppressed the production of mesothelin, OCT4, and SOX 2 but recovered the expression of TAp63 in flagellin- or imiquimod-treated SKOV3 cells. Our results suggest that the PI3K/WAVE3 signaling pathway initiates the invasion and metastasis of ovarian cancer after stimulation with TLR5/7.

The expression of p63 plays an important role in the generation of epithelial tissues during the developmental stages of both humans and murine animals (39). Furthermore, the disruption of p63 results in more aggressive and metastatic tumors through the upregulation of genes associated with increased invasiveness and metastasis (40). TLR4 stimulation had been shown to promote the invasive activity of colon cancer cells by TAp63-mediated GSK-3 $\beta$ activation (25). Moreover, the loss of p63 expression accelerates the metastatic spread and aggressive invasion of the cancer cells (41). In this study, the TLR5/7-stimulated ovarian cancer cells also showed reduced migratory activities along with the suppression of TAp63 expression. These results suggest that the role of TAp63 in cancer metastasis is still controversial and cell-type dependent.

The expression of embryonic stem cell markers, including SOX2 and OCT4 is significantly associated with the progression of various human malignancies (42). SOX2 promotes the metastasis of breast and prostate cancer cells by promoting epithelial-to-mesenchymal transition (EMT) (43). Silencing of 
the p63 gene using siRNA suppresses the expression of SOX2 and OCT4 in mouse embryonic fibroblasts (44). Although the stimulation with flagellin or imiquimod of SKOV3 cells induced the expression of SOX 2 and OCT4 in this study, the activation of TAp63 was significantly reduced. Conversely, the knockdown of WAVE3 attenuated mesothelin, OCT4, and SOX2 production, whereas the expression of TAp63 was recovered. In addition, the targeted inhibition of mesothelin recovered the expression of TAp63 but had no effect on the production of OCT4 and SOX2 in the flagellin- or imiquimodtreated SKOV3 cells.

TLR stimulation is closely associated with carcinogenesis and progression as well as the production of various cytokines and chemokines (3). In this study, we also identified the critical role of TLR5/7-mediated WAVE3 signaling in the invasion and metastasis of ovarian cancer cells in the independent regulation of expression of mesothelin/CA125 and OCT4 and SOX2. Therefore, these results suggest that WAVE3 might be a promising candidate for the development of a new targeted therapy against ovarian cancer metastasis.

\section{Acknowledgements}

This study was supported by the Basic Science Research Program of Ministry of Education (NRF-2015R1D1A1A01056672) and Ministry of Science, ICT and Future Planning (NRF-2015R1C1A2A01053732) through the National Research Foundation (NRF) of Republic of Korea.

\section{References}

1. Brun JL, Feyler A, Chêne G, Saurel J, Brun G and Hocké C: Long-term results and prognostic factors in patients with epithelial ovarian cancer. Gynecol Oncol 78: 21-27, 2000.

2. Chaffer CL and Weinberg RA: A perspective on cancer cell metastasis. Science 331: 1559-1564, 2011.

3. Rakoff-Nahoum S and Medzhitov R: Toll-like receptors and cancer. Nat Rev Cancer 9: 57-63, 2009.

4. Kim S, Takahashi H, Lin WW, Descargues P, Grivennikov S, Kim Y, Luo JL and Karin M: Carcinoma-produced factors activate myeloid cells through TLR2 to stimulate metastasis. Nature 457: 102-106, 2009

5. Harmey JH, Bucana CD, Lu W, Byrne AM, McDonnell S, Lynch C, Bouchier-Hayes D and Dong Z: Lipopolysaccharideinduced metastatic growth is associated with increased angiogenesis, vascular permeability and tumor cell invasion. Int J Cancer 101: 415-422, 2002.

6. Merrell MA, Ilvesaro JM, Lehtonen N, Sorsa T, Gehrs B, Rosenthal E, Chen D, Shackley B, Harris KW and Selander KS Toll-like receptor 9 agonists promote cellular invasion by increasing matrix metalloproteinase activity. Mol Cancer Res 4 : 437-447, 2006

7. Zhou M, McFarland-Mancini MM, Funk HM, Husseinzadeh N, Mounajjed T and Drew AF: Toll-like receptor expression in normal ovary and ovarian tumors. Cancer Immunol Immunother 58: 1375-1385, 2009.

8. Chang K and Pastan I: Molecular cloning of mesothelin, a differentiation antigen present on mesothelium, mesotheliomas, and ovarian cancers. Proc Natl Acad Sci USA 93: 136-140, 1996.

9. Chang MC, Chen CA, Chen PJ, Chiang YC, Chen YL, Mao TL, Lin HW, Lin Chiang WH and Cheng WF: Mesothelin enhances invasion of ovarian cancer by inducing MMP-7 through MAPK/ ERK and JNK pathways. Biochem J 442: 293-302, 2012.

10. Servais EL, Colovos C, Rodriguez L, Bograd AJ, Nitadori J, Sima C, Rusch VW, Sadelain M and Adusumilli PS: Mesothelin overexpression promotes mesothelioma cell invasion and MMP-9 secretion in an orthotopic mouse model and in epithelioid pleural mesothelioma patients. Clin Cancer Res 18: 2478-2489, 2012
11. Rump A, Morikawa Y, Tanaka M, Minami S, Umesaki N, Takeuchi M and Miyajima A: Binding of ovarian cancer antigen CA125/MUC16 to mesothelin mediates cell adhesion. J Biol Chem 279: 9190-9198, 2004.

12. Markman M, Federico M, Liu PY, Hannigan E and Alberts D: Significance of early changes in the serum CA-125 antigen level on overall survival in advanced ovarian cancer. Gynecol Oncol 103: 195-198, 2006.

13. Chen SH, Hung WC, Wang P, Paul C and Konstantopoulos K: Mesothelin binding to CA125/MUC16 promotes pancreatic cancer cell motility and invasion via MMP-7 activation. Sci Rep 3: 1870, 2013.

14. Bharadwaj U, Marin-Muller C, Li M, Chen C and Yao Q: Mesothelin confers pancreatic cancer cell resistance to TNF- $\alpha$ induced apoptosis through $\mathrm{Akt} / \mathrm{PI} 3 \mathrm{~K} / \mathrm{NF}-\mathrm{\kappa B}$ activation and IL-6/Mcl-1 overexpression. Mol Cancer 10: 106, 2011.

15. Osaki M, Oshimura M and Ito H: PI3K-Akt pathway: Its functions and alterations in human cancer. Apoptosis 9: 667-676, 2004.

16. Yu L and Chen S: Toll-like receptors expressed in tumor cells: Targets for therapy. Cancer Immunol Immunother 57: 1271-1278, 2008.

17. Park GB, Chung YH and Kim D: Induction of galectin-1 by TLR-dependent PI3K activation enhances epithelial-mesenchymal transition of metastatic ovarian cancer cells. Oncol Rep 37: 3137-3145, 2017.

18. Suetsugu S and Takenawa T: Regulation of cortical actin networks in cell migration. Int Rev Cytol 229: 245-286, 2003

19. Suetsugu S, Yamazaki D, Kurisu S and Takenawa T: Differential roles of WAVE1 and WAVE2 in dorsal and peripheral ruffle formation for fibroblast cell migration. Dev Cell 5: 595-609, 2003.

20. Kulkarni S, Augoff K, Rivera L, McCue B, Khoury T, Groman A, Zhang L, Tian L and Sossey-Alaoui K: Increased expression levels of WAVE3 are associated with the progression and metastasis of triple negative breast cancer. PLoS One 7: e42895, 2012.

21. Ji Y, Li B, Zhu Z, Guo X, He W, Fan Z and Zhang W: Overexpression of WAVE3 promotes tumor invasiveness and confers an unfavorable prognosis in human hepatocellular carcinoma. Biomed Pharmacother 69: 409-415, 2015.

22. Moazzam M, Ye L, Sun PH, Kynaston H and Jiang WG: Knockdown of WAVE3 impairs HGF induced migration and invasion of prostate cancer cells. Cancer Cell Int 15: 51, 2015.

23. Sossey-Alaoui K, Li X, Ranalli TA and Cowell JK: WAVE3mediated cell migration and lamellipodia formation are regulated downstream of phosphatidylinositol 3-kinase. J Biol Chem 280: 21748-21755, 2005 .

24. Rutkowski MR, Stephen TL, Svoronos N, Allegrezza MJ, Tesone AJ, Perales-Puchalt A, Brencicova E, Escovar-Fadul X, Nguyen JM, Cadungog MG, et al: Microbially driven TLR5dependent signaling governs distal malignant progression through tumor-promoting inflammation. Cancer Cell 27: 27-40, 2015.

25. Park GB, Chung YH, Gong JH, Jin DH and Kim D: GSK-3 $\beta$ mediated fatty acid synthesis enhances epithelial to mesenchymal transition of TLR4-activated colorectal cancer cells through regulation of TAp63. Int J Oncol 49: 2163-2172, 2016.

26. Vergara D, Merlot B, Lucot JP, Collinet P, Vinatier D, Fournier I and Salzet M: Epithelial-mesenchymal transition in ovarian cancer. Cancer Lett 291: 59-66, 2010.

27. Beaufort CM, Helmijr JC, Piskorz AM, Hoogstraat M, RuigrokRitstier K, Besselink N, Murtaza M, van IJcken WF, Heine AA, Smid M, et al: Ovarian cancer cell line panel (OCCP): Clinical importance of in vitro morphological subtypes. PLoS One 9: e103988, 2014

28. Leis O, Eguiara A, Lopez-Arribillaga E, Alberdi MJ, HernandezGarcia S, Elorriaga K, Pandiella A, Rezola R and Martin AG: Sox 2 expression in breast tumours and activation in breast cancer stem cells. Oncogene 31: 1354-1365, 2012.

29. Kumar SM, Liu S, Lu H, Zhang H, Zhang PJ, Gimotty PA, Guerra M, Guo W and Xu X: Acquired cancer stem cell phenotypes through Oct4-mediated dedifferentiation. Oncogene 31: 4898-4911, 2012.

30. Kelly MG, Alvero AB, Chen R, Silasi DA, Abrahams VM, Chan S, Visintin I, Rutherford T and Mor G: TLR-4 signaling promotes tumor growth and paclitaxel chemoresistance in ovarian cancer. Cancer Res 66: 3859-3868, 2006.

31. Cheng WF, Huang CY, Chang MC, Hu YH, Chiang YC, Chen YL, Hsieh CY and Chen CA: High mesothelin correlates with chemoresistance and poor survival in epithelial ovarian carcinoma. Br J Cancer 100: 1144-1153, 2009. 
32. Bast RC Jr: Status of tumor markers in ovarian cancer screening. J Clin Oncol 21 (Suppl 10): 200-205, 2003.

33. Shimizu A, Hirono S, Tani M, Kawai M, Okada K, Miyazawa M, Kitahata Y,Nakamura Y,Noda T, Yokoyama S, et al: Coexpression of MUC16 and mesothelin is related to the invasion process in pancreatic ductal adenocarcinoma. Cancer Sci 103: 739-746, 2012.

34. Millard TH, Sharp SJ and Machesky LM: Signalling to actin assembly via the WASP (Wiskott-Aldrich syndrome protein)family proteins and the Arp2/3 complex. Biochem J 380: 1-17, 2004.

35. Miki H, Suetsugu S and Takenawa T: WAVE, a novel WASPfamily protein involved in actin reorganization induced by Rac. EMBO J 17: 6932-6941, 1998.

36. Sossey-Alaoui K, Ranalli TA, Li X, Bakin AV and Cowell JK: WAVE3 promotes cell motility and invasion through the regulation of MMP-1, MMP-3, and MMP-9 expression. Exp Cell Res 308: 135-145, 2005

37. Davuluri G, Augoff K, Schiemann WP, Plow EF and SosseyAlaoui K: WAVE3-NFkB interplay is essential for the survival and invasion of cancer cells. PLoS One 9: e110627, 2014.

38. Cantley LC: The phosphoinositide 3-kinase pathway. Science 296: 1655-1657, 2002

39. Yang A, Kaghad M, Wang Y, Gillett E, Fleming MD, Dötsch V, Andrews NC, Caput D and McKeon F: p63, a p53 homolog at $3 q 27-29$, encodes multiple products with transactivating, deathinducing, and dominant-negative activities. Mol Cell 2: 305-316, 1998.
40. Koga F, Kawakami S, Fujii Y, Saito K, Ohtsuka Y, Iwai A, Ando N, Takizawa T, Kageyama Y and Kihara K: Impaired p63 expression associates with poor prognosis and uroplakin III expression in invasive urothelial carcinoma of the bladder. Clin Cancer Res 9: 5501-5507, 2003

41. Barbieri CE, Tang LJ, Brown KA and Pietenpol JA: Loss of p63 leads to increased cell migration and up-regulation of genes involved in invasion and metastasis. Cancer Res 66: 7589-7597, 2006.

42. Luo W, Li S, Peng B, Ye Y, Deng X and Yao K: Embryonic stem cells markers SOX2, OCT4 and Nanog expression and their correlations with epithelial-mesenchymal transition in nasopharyngeal carcinoma. PLoS One 8: e56324, 2013.

43. Li X, Xu Y, Chen Y, Chen S, Jia X, Sun T, Liu Y, Li X, Xiang R and Li N: SOX2 promotes tumor metastasis by stimulating epithelialto-mesenchymal transition via regulation of $\mathrm{WNT} / \beta$-catenin signal network. Cancer Lett 336: 379-389, 2013.

44. Alexandrova EM, Petrenko O, Nemajerova A, Romano RA, Sinha $S$ and Moll UM: $\triangle$ Np63 regulates select routes of reprogramming via multiple mechanisms. Cell Death Differ 20: 1698-1708, 2013. 